\title{
Análisis de la Tolerancia al Ruido de Características Basadas en Dinámica no Lineal Sobre Señales Fonocardliográficas
}

\author{
Carolina Ospina-Aguirre ${ }^{1}$ \\ Jorge A. Gómez-García ${ }^{2}$ \\ Edilson Delgado-Trejos ${ }^{3}$ \\ Germán Castellanos-Domínguez ${ }^{4}$
}

\section{Resumen}

Una propiedad muy deseable en sistemas de detección automática de patologías es la robustez ante el ruido, tal que su presencia no afecte notablemente la habilidad de detectar patologías. El presente trabajo estudia la capacidad discriminatoria que características de la dinámica no lineal, en particular, el Exponente de Hurst, el Máximo Exponente de Lyapunov y la Dimensión de Correlación, puedan proveer en la detección de soplos cardíacos usando señales fonocardiográficas contaminadas con diferentes niveles de ruido. A su vez se hacen comparaciones con características obtenidas a partir de representaciones tiempo frecuencia. Los resultados muestran la fortaleza de las características basadas en dinámica no lineal para tareas de clasificación de estados funcionales, aún sobre señales con altos niveles de ruido.

1 Grupo de Control y Procesamiento Digital de Señales, Universidad Nacional de Colombia sede Manizales, cospinaa@unal.edu.co

2 Grupo de Control y Procesamiento Digital de Señales, Universidad Nacional de Colombia sede Manizales, jorgomezg@unal.edu.co

3 Centro de Investigación, Grupo MIRP, INSTITUTO TECNOLÓGICO METROPOLITANO, edilsondelgado@itm.edu.co

4 Grupo de Control y Procesamiento Digital de Señales, Universidad Nacional de Colombia sede Manizales, cgcastellanosd@unal.edu.co

Fecha de recepción: 24 de Agosto de 2010

Fecha de aceptación: 26 de Octubre de 2010 
[190] Análisis de la Tolerancia al Ruido de Características Basadas en Dinámica no Lineal Sobre Señales Fonocardiográficas

\section{Palabras clave}

Exponente de Hurst, FCG, máximo exponente de Lyapunov, relación señal ruido, técnicas de dinámica no lineal.

\section{Abstract}

A very desirable attribute in automatic pathology detection systems is noise robustness, such that the presence of noise should not significantly affect the ability to detect pathologies. This paper explores the discriminatory potential that nonlinear dynamic features, in particular, the Hurst exponent, the maximum Lyapunov exponent and correlation dimension, can provide in the detection of heart murmurs using phonocardiographic signals contaminated with different levels of noise. At the same time comparing with features obtained from time frequency representations. The results show the strength of features based on nonlinear dynamics for functional state classification tasks, even on signals with high noise levels.

\section{Keywords}

Hurst exponent, largest Lyapunov exponent, nonlinear dynamics techniques, PCG, SNR. 


\section{INTRODUCCIÓN}

Se ha demostrado que las tareas de reconocimiento de patrones, orientadas al diagnóstico automatizado de patologías, pueden ser alteradas por perturbaciones que contaminan la señal y disminuyen la efectividad en clasificación (Messer et al., 2001). Propiamente sobre las señales Fonocardiográficas (FCG), se presentan varias fuentes de ruido contaminante, siendo una de las mayores, la generada por los sonidos respiratorios en el momento de la adquisición de los registros. Debido a esto, es importante encontrar características que sean capaces de presentar resultados confiables en la detección de patologías aún bajo la influencia de fuertes perturbaciones.

La demostrada presencia de comportamiento no lineal en señales FCG (Nigam et al., 2005), y los previos resultados logrados con caracterizaciones no lineales (Delgado, Castellanos \& Vallverdú, 2009), hacen pensar que un análisis con este tipo de características puede ser producente en tareas de detección de patologías en señales contaminadas por ruido.

Por otra parte, cambios significativos en condiciones fisiológicas pueden producir variaciones relevantes en las características de las bioseñales, entendidas como no estacionariedad de las mismas. Con el fin de analizar tales cambios, representaciones tiempo-frecuencia son requeridas (Tarvainen et al., 2009). Debido a esto, durante las últimas décadas representaciones tiempo-frecuencia han prevalecido en el análisis de señales no estacionarias, en particular FCG (Tarvainen et al., 2009; Quiceno et al., 2009; Seidic et al., 2009; Avendaño et al., 2010).

En este trabajo se pretende estudiar la inmunidad al ruido que características tiempo frecuencia y basadas en dinámica no lineal presentan en labores relacionadas con reconocimiento de patrones. La etapa de análisis con técnicas de dinámica no lineal propone la utilización de las 3 medidas de complejidad más comunes: Dimensión de correlación (D2), el Exponente de Lyapunov (LLE) y el Exponente de Hurst (H), en labores de detección tanto de soplos sistólicos como diastólicos en señales FCG con diferentes cantidades de SNR (Signal-Noise Ratio). Será evaluada a su vez la 
eficiencia en clasificación que características calculadas a partir de la TFR como la Energía instantánea, Frecuencia instantánea, Ancho de banda, vectores propios, Centroides espectrales y Coeficientes cepstrales, pueden proveer.

\section{MATERIALES Y MÉTODOS}

\subsection{Base de Datos}

La base de datos utilizada en este estudio se compone de 148 registros de personas adultas. Se utilizó un estetoscopio electrónico (WelchAllyn modelo Meditron) para adquirir los sonidos del corazón simultáneamente, con un estándar de 3 derivaciones (la derivación DII fue utilizada como una referencia de tiempo debido a que el complejo QRS se define claramente). Ambas señales se digitalizaron a $44,1 \mathrm{kHz}$ con 16 bits por muestra. Una serie de 50 pacientes fueron catalogados como normales, mientras que 98 han sido etiquetados como soplos cardíacos, causados por trastornos de la válvula (estenosis aórtica, insuficiencia mitral, etc.). Fue necesario realizar el diagnóstico en cada latido y no en el conjunto de señales FCG adquiridas para cada paciente, entonces, 360 latidos individuales fueron extraídos, 180 de cada clase. Los latidos individuales fueron seleccionados como los mejores de cada señal, después de una inspección visual y sonora por un cardiólogo, con lo que se descartó la presencia de ruido por artefactos o de cualquier otro tipo que pudiera perjudicar el rendimiento de los algoritmos (Delgado et al., 2009).

\subsection{Metodología Propuesta}

En la Fig. 1 se muestran las etapas de la metodología implementada en el presente trabajo. 


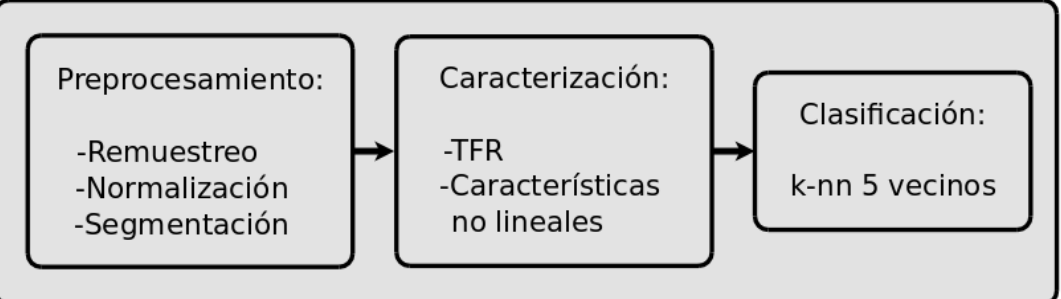

Fig. 1. Metodología de experimentación

\subsection{Preprocesamiento}

En esta etapa las señales fueron remuestreadas a $4 \mathrm{kHz}$ según lo sugerido por Quiceno et al. (2009), centralizadas, y después normalizadas de acuerdo a lo dado por (1)

$$
Y=\frac{2 y_{c}}{\max \left(y_{c}\right)-\min \left(y_{c}\right)}
$$

donde $y_{c}$ es la señal centralizada. Posteriormente, la señal es segmentada de acuerdo con la metodología propuesta en Delgado et al. (2009), de forma que quedan separados los sonidos $S_{1}$ y $S_{2}$, y los silencios de sístole y de diástole en cada una de las señales.

\subsection{Caracterización}

En esta etapa se propone el uso de dos tipos de características: las derivadas de dinámica no lineal y las obtenidas a partir de las de representación tiempo-frecuencia (TFR).

\subsubsection{Dinámica no lineal}

Se requiere una etapa previa a la caracterización con técnicas de dinámica no lineal en la que se lleve la serie de tiempo a un espacio $m$ - dimensional llamado espacio de fases o de estados. Esta reconstrucción es lograda gracias a un proceso llamado embebimiento, que se define para una serie de tiempo $x=\left\{x_{1}, x_{2}, \ldots, x_{n}\right\}$, los vectores de estado reconstruidos como en (2) 
$\mathbf{X}(t)=[x(t), x(t-\tau), x(t-2 \tau), \ldots, x(t-(m-1) \tau]$

donde, $m$ es la dimensión de embebimiento y $\tau$ es el tiempo de retardo, ambos parámetros se denominan parámetros de embebimiento o de reconstrucción del atractor en el espacio de estados. A continuación se realiza la caracterización con dinámica no lineal mediante los parámetros: Máximo Exponente de Lyapunov, Dimensión de Correlación y Exponente de Hurst.

\subsubsection{Máximo Exponente de Lyapunov (LLE)}

Informa sobre el nivel de impredecibilidad de la serie de tiempo $x=\left\{x_{1}, x_{2}, \ldots, x_{n}\right\}$. Si la serie de tiempo es la respuesta de un sistema caótico, las trayectorias del atractor en el espacio de estados evolucionan temporalmente y se presenta una separación entre las mismas que crece a una tasa exponencial (Delgado et al., 2009).

\subsubsection{Dimensión de correlación (D2)}

Este parámetro estima la dimensión fractal del atractor en el espacio de estados, con lo que cuantifica en una dimensión la autosimilaridad de una serie de tiempo embebida (Kantz, 2004).

\subsubsection{Exponente de Hurst $(H)$}

Este parámetro cuantifica la naturaleza dinámica de la serie de tiempo y permite ponderar hasta qué punto los datos pueden ser representados por un movimiento de tipo browniano. Puede verse como una medida de la persistencia del proceso a seguir tendencias o de la correlación existente entre diferentes puntos en la serie de tiempo (Sprott, 2003).

\subsubsection{Representación Tiempo Frecuencia (TFR)}

La representación tiempo frecuencia está basada en el principio de incertidumbre, donde la frecuencia de la señal en un 
tiempo particular no puede ser determinada. A veces, ambos parámetros son necesarios (tiempo y frecuencia), especialmente en señales no estacionarias con cambios dinámicos. De esta manera, la transformada corta de Fourier (notada como STFR por sus siglas en inglés), introduce un concepto de localización, utilizando una ventana de reducción $\varphi$. El espectrograma es una TFR común, utilizada en diferentes aplicaciones, y calculada como el cuadrado de la magnitud del STFT, dado por (3)

$|\langle x, \phi\rangle|=\left|\int_{\mathrm{T}} x(\tau) \phi(\tau-t) e^{-j 2 \pi f \tau} d \tau\right|^{2} \triangleq \mathbf{S}_{x}(t, f)$

\subsection{Clasificación}

Se propone el uso del clasificador basado en los $K$ vecinos más cercanos, ajustando el valor de $K$ en 5 vecinos como se propuso en (Quiceno et al., 2009) usando Cross-validación 10-folds con el 70\% de las muestras para entrenamiento y el $30 \%$ restante para validación.

\section{RESULTADOS}

La Fig. 2 muestra la densidad espectral de potencia para una señal fonocardiográfica original y contaminada por ruido, en (a) para $\mathrm{SNR}=1$ y en (b) para $\mathrm{SNR}=50$. Nótese que cuanto mayor es la SNR, la diferencia de área entre la densidad espectral de potencia de la señal original y la contaminada por ruido es menor. La Tabla 1 muestra los valores porcentuales de la diferencia del área entre las densidades espectrales de potencia para diferentes valores de SNR.

Debido al alto costo computacional de la caracterización de la base de datos, se eligieron los valores SNR de la tabla que generaron un cambio porcentual considerable en la diferencia del área entre las densidades espectrales de las señales originales y las contaminadas. Esto es: SNR 1, 10, 25 y 50. 
[196] Análisis de la Tolerancia al Ruido de Características Basadas en Dinámica no Lineal Sobre Señales Fonocardiográficas

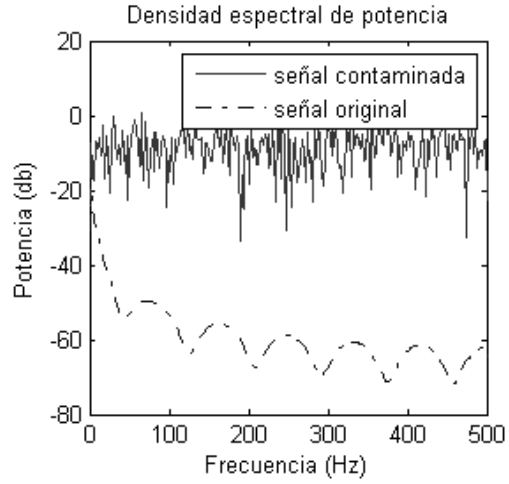

a)

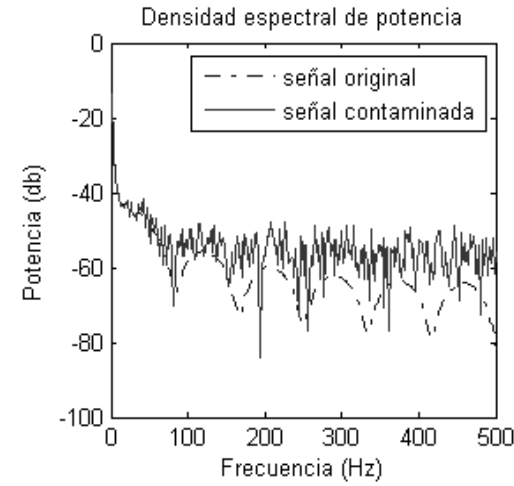

b)

Fig. 2. Densidad espectral de potencia para la señal original y la contaminada con ruido: (a) $\mathrm{SNR}=1$ (b) $\mathrm{SNR}=50$

Tabla 1. Diferencia de área entre la densidad espectral de potencia de la señal original y la señal con ruido, para diferentes valores de SNR por clase

\begin{tabular}{ccc}
\hline SNR & Normales & Patológicas \\
\hline 1 & 88,00 & 86,75 \\
5 & 82,40 & 80,60 \\
10 & 68,34 & 65,10 \\
20 & 61,35 & 57,42 \\
25 & 54,37 & 49,72 \\
30 & 54,37 & 49,74 \\
35 & 54,37 & 49,73 \\
40 & 54,38 & 49,73 \\
45 & 54,38 & 49,72 \\
50 & 54,35 & 49,70 \\
\hline
\end{tabular}

En la Fig. 3 se observa la dispersión de las características calculadas sobre el segmento sístole sin agregar ruido (3a) y con diferentes valores de SNR: SNR 25 (3b), SNR 10 (3c), SNR 1 (3d).

La Tabla 2 muestra los porcentajes de acierto usando características no lineales, para los segmentos sístole y diástole para los valores de SNR elegidos. La Tabla 3 muestra los resultados para las características extraídas de las TFR. 


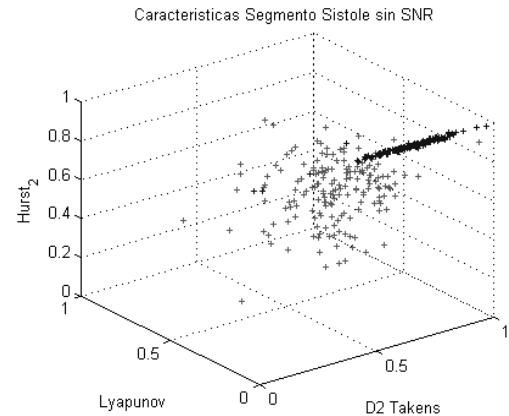

a)

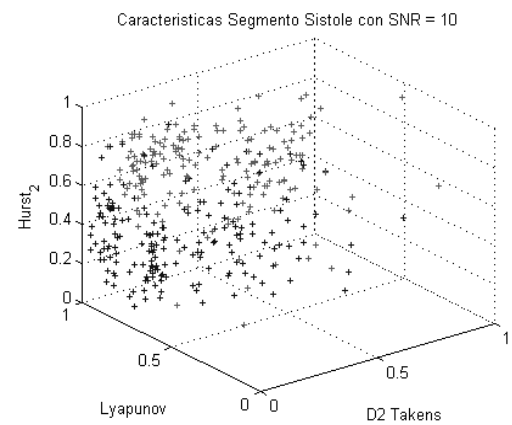

c)

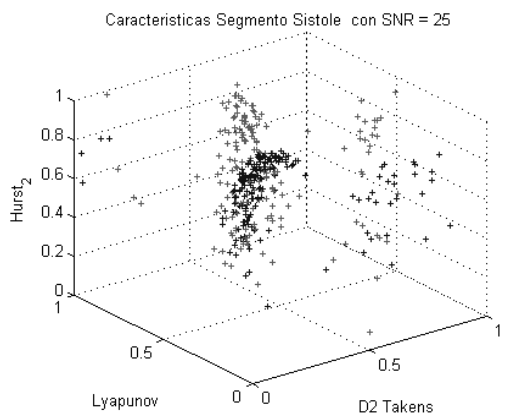

b)

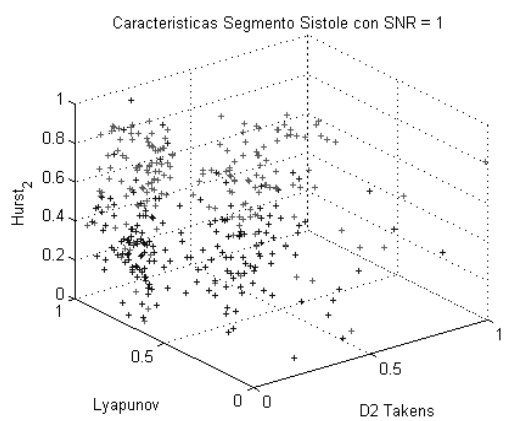

d)

Fig. 3. Dispersión de las características de complejidad calculadas sobre el segmento sístole

Tabla 2. Porcentajes de acierto sobre segmentos sístole y diástole para diferentes valores de SNR con características no lineales

\begin{tabular}{ccc}
\hline SNR & $\begin{array}{c}\text { Porcentaje de acierto, } \\
\text { sístole }\end{array}$ & $\begin{array}{c}\text { Porcentaje de acierto, } \\
\text { diástole }\end{array}$ \\
\hline 1 & 82,5 & 81,3 \\
10 & 84,7 & 81,7 \\
25 & 88,0 & 90,8 \\
50 & 96,1 & 97,2 \\
-- & 97,4 & 98,9 \\
\hline
\end{tabular}


Tabla 3. Porcentajes de acierto sobre segmentos sístole y diástole para diferentes valores de SNR a partir de las TFR

\begin{tabular}{ccc}
\hline SNR & $\begin{array}{c}\text { Porcentaje de acierto, } \\
\text { sístole }\end{array}$ & $\begin{array}{c}\text { Porcentaje de acierto, } \\
\text { diástole }\end{array}$ \\
\hline 1 & 81,4 & 76,4 \\
10 & 86,3 & 77,3 \\
25 & 86,8 & 77,3 \\
50 & 90,1 & 80,5 \\
-- & 96,9 & 90,9 \\
\hline
\end{tabular}

\section{DISCUSIÓN Y CONCLUSIONES}

Las características basadas en dinámica no lineal, muestran en términos generales, un buen desempeño en tareas clasificatorias, a pesar de una relativa pérdida en acierto producto de la influencia del ruido agregado.

La Fig. 3 ilustra este efecto, mostrando la evolución de la dispersión entre clases a medida que aumenta el SNR. Se puede observar como la separabilidad aparente entre clases disminuye a mayor ruido, sin que esto sin embargo logre empañar el buen desempeño dado por el sistema de clasificación. Así, en la figura $3 \mathrm{~d}$ se ven nubes poco dispersas que podrían considerarse separables. A medida que el SNR disminuye, las nubes de puntos van dispersándose hasta que ambas clases tienden a juntarse. Sin embargo, aún en casos de adición fuerte de ruido, las tasas de acierto clasificatorio resultantes se pueden considerar aceptables, haciendo pensar a la luz de los resultados, en la robustez ante el ruido de las características basadas en dinámica no lineal.

De otra parte, las características basadas en TFR mostraron desempeños menores en clasificación a los dados por las características no lineales para todos los casos de análisis.

Después de proceder a agregar una fuerte cantidad de ruido al sistema, se lograron tasas que no son consideradas muy aceptables. Cuando la adición de ruido fue pequeña se observó a su vez una baja considerable en desempeños al compararla con las señales sin contaminación. Tales resultados hacen pensar en el poco nivel de robustez ofrecido por este tipo de características y el 
mayor acierto que es posible de lograr con las características no lineales.

Los resultados dejan concluir entonces, que las características no lineales son más robustas al ruido que las características TFR, a pesar de que ambas técnicas son ampliamente usadas en sistemas no estacionarios con presencia de perturbaciones, mostrándose también que las medidas no lineales son capaces de mantener un desempeño correcto en labores de reconocimiento de patrones con señales FCG contaminadas por ruido, en la detección de soplos sistólicos y diastólicos.

\section{AGRADECIMIENTOS}

Este trabajo se enmarca en el proyecto "Servicio de monitoreo remoto de actividad cardiaca para el tamizaje clínico en la red de telemedicina del Departamento de Caldas" financiado por Proyectos de investigación e innovación conjuntos entre grupos de trabajo académico, Universidad de Caldas y Universidad Nacional Sede Manizales, hacia tercer milenio. Adicionalmente, los autores quieren agradecen al programa Jóvenes Investigadores e Innovadores p-2008-0781, del Instituto Colombiano para el Desarrollo de la Ciencia y la Tecnología Colciencias. Igualmente, este trabajo es resultado de investigación del proyecto P09225, titulado "Metodología dinámica de detección de soplos cardiacos basado en Hidden Markov Models" y financiado por el INSTITUTO TECNOLÓGICO METROPOLITANO - ITM. Se presenta un agradecimiento especial al investigador Diego L. Guarín por sus comentarios y sugerencias que constituyeron un gran aporte a este trabajo.

\section{REFERENCIAS}

Avendaño, L.D, Godino, J.I., Blanco, M., Castellanos, C.G., (2010); Feature Extraction from Parametric Time-Frequency Representations for Heart Murmur Detection. Annals of Biomedical Engineering. 38(8), 2716-2732. 
Delgado, E., Castellanos, C.G., Vallverdú, M., (2009); Análisis de Relevancia en Espacios de Representación Orientado al Soporte de Diagnóstico: Aplicaciones en la Dinámica Cardíaca. Manizales: Publicaciones Universidad Nacional de Colombia.

Kantz, T., Schreiber, T., (2004); Nonlinear Time Series Analysis. University of Cambridge.

Messer, S., Agzarian, J., Abbott, D., (2001); Optimal wavelet denoising for phonocardiograms. Microelectronics Journal. 931-941.

Nigam, V., Priemer, R., (2005); Accessing heart dynamics to estimate durations of heart sound. Physiol Meas. 1005-1018.

Quiceno, A.F., Godino, J.I., Blanco, M., Castellanos, C.G., (2009); Selection of Dynamic Features Based on Time-Frequency Representations for Heart Murmur Detection from Phonocardiographic Signals. Annals of Biomedical Engineering. 38(1), 118-137.

Sejdic, E., Djurovic, I., Jiang, J., (2009); Time-frequency feature representation using energy concentration: an overview of recent advances. Digital Signal Process. 19(1), 153-183.

Sprott, J.C., (2003); Chaos and time-series analysis. Oxford University Press.

Tarvainen, M.P., Georgiadis, S., Lipponen, J.A., Hakkarainen, M., Karjalainen, P.A., (2009); Time-varying spectrum estimation of heart rate variability signals with Kalman smoother algorithm. Engineering in Medicine and Biology Society. EMBC 2009. Annual International Conference of the IEEEand time-series analysis. Oxford University Press. 